Check for updates

Cite this: RSC Adv., 2018, 8, 9963

\title{
Self-assembly of a nano hydrogel colloidal array for the sensing of humidity
}

\begin{abstract}
Zhe Wang, (D) a Min Xue, ${ }^{\text {*a }}$ Herong Zhang, ${ }^{a}$ Zihui Meng, ${ }^{\text {*a }}$ Kenneth J. Shea, ${ }^{\mathrm{b}}$ Lili Qiu, $^{a}$ Tiantian $\mathrm{Ji}^{\mathrm{a}}$ and Tengsheng $\mathrm{Xie}^{\mathrm{a}}$

Traditional artificial opals are assembled from silica or polystylene colloidals which have poor swellability and a lower response to stimuli. A novel three-dimensional photonic crystal array sensor which has a high stability and desired structural colour was fabricated from the self assembly of nano hydrogel colloids. The nano hydrogel colloids were prepared by co-polymerisation of $N$-isopropylacrylamide, functional monomer acrylic acid and $N$-tert-butylacrylamide. The relative humidity from $20 \%$ to $100 \%$ could be detected rapidly via the reflection spectrum of the nano hydrogel colloidal array with a maximum amount of red shift of $24 \mathrm{~nm}$. The response kinetics for humidity of the nano hydrogel colloidal array were investigated, and correspondingly, a rational response mechanism of the compactness of the close-packed structure caused by the swelling of the nano hydrogel colloidal array was discussed. The nano hydrogel colloidal array sensor presented good reversibility and can be reused for at least five rounds.
\end{abstract}

Received 21st November 2017 Accepted 13th February 2018

DOI: 10.1039/c7ra12661a

rsc.li/rsc-advances construct PC from mono-dispersed latex spheres. ${ }^{28,29}$ However, photonic crystal arrays fabricated by the self-assembly of solid spheres, are difficult to respond to external stimuli by deformation for their small swelling ability and low mass transfer. The common practice of realizing lattice spacing variations in photonic crystal arrays is incorporating responsive gel into photonic crystal arrays to fabricate the opal structure. Smith et al. developed an air-stable two-dimension opal PC sensor which was immersed into ethylguanidine perchlorate ionic liquid for visual detection of vapour and ammonia because of the negligible ionic liquid vapour pressure. ${ }^{30}$ Barry et al. developed a polyacrylamide inverse opal hydrogel to detect ambient humidity variations through an optical change. ${ }^{31}$ However, the photonic crystal bandgap after embedding will change due to the filling of the gel medium. Moreover, the filling process often results in damage to the array structure, which requires the operator to be extremely careful.

Soft materials can also be used for the preparation of photonic crystal arrays. Compared to the solid spheres, soft materials ${ }^{32}$ with a larger swelling ratio and exposed functional groups on the surface of the particles are easier to respond to external stimuli. Recently, a kind of novel nano hydrogel colloidal (NHC) material with sensing, processing and executive function that hold great promise in nanotechnology for diagnostics, drug delivery, protein separation and bioinspired designing was synthesized as polymer hydrogel colloidal nanoparticles. ${ }^{33}$ The NHC has the similar composition of hydrogel nanoparticles that is called plastic antibody. ${ }^{34}$ It is capable of combining with target biomolecules for its good biocompatibility and affinity, and can be used as a substitute for 
antibody. It has been applied in toxins elimination successfully in vivo and in vitro. The responsive NHCs are a class of polymers which undergoing a volume phase transition in response to minimal environmental stimulus. Besides, temperatureresponsive colloidal nanoparticles containing the spatial network structure have a remarkable capacity to adsorb water. There are also substantial hydrophilic groups and hydrophobic groups at side chain, which makes the polymers sensitive to water. In addition, the preparation process of the threedimensional NHCA is easier than that of the opal or inverse opal PC hydrogel film. However, PC colloidal arrays that was assembled with NHC is rarely reported.

In this study, we focused on the PC array fabricated by hydrogel nanoparticles for the response of humidity. We innovatively constructed a PC sensor fabricated from soft and biofriendly NHC, and investigated its response to humidity. To a certain extent, this work expanded the materials that could be used for the preparation of photonic crystal. Furthermore, the nano hydrogels have been verified to be capable of binding to specific targets including peptides and proteins, ${ }^{34,35}$ which provides the possibility of recognizing biological macromolecules using the as-prepared NHCA in the follow-up study.

\section{Experimental section}

\section{Materials}

$N$-Isopropylacrylamide (NIPAM), acrylic acid (AAc), and $N, N^{\prime}$ methylenebisacrylamide (BIS) were purchased from J\&K Chemicals Co. Ltd.; $N$-tert-butylacrylamide ( $t$-BAM) was from Aladdin Chemicals Co. Ltd.; sodium dodecylsulfate (SDS) was from Fuchen chemical reagent factory; ammonium persulfate (APS) was from Beijing Chemical Reagent Co. Ltd. All chemicals were used as received, except that NIPAM was recrystallized by hexane before usage. Water used in the experiment was distilled and purified by an Aquapro purification system. Glass slides $(50 \times 24 \mathrm{~mm}$ and $24 \times 24 \mathrm{~mm})$ were purchased from Weiss Experiment Products Co. Ltd. and treated with a $\mathrm{H}_{2} \mathrm{SO}_{4} / \mathrm{H}_{2} \mathrm{O}_{2}$ $(7 / 3, v / v)$ solution for $24 \mathrm{~h}$.

\section{Synthesis and characterization of nano hydrogel colloids}

The nano hydrogel colloids (NHC) were synthesized using precipitation polymerization method. ${ }^{36}$ NiPAM $(139.7 \mathrm{mg})$, AAc (46.8 mg), $t$-BAM (165.3 mg), BIS (10 mg) and SDS were dissolved in $50 \mathrm{~mL}$ water of a total volume. Then the initiator APS $(30 \mathrm{mg})$ was added after the solution degassed by nitrogen for $30 \mathrm{~min}$. The polymerization reaction was carried out at $65^{\circ} \mathrm{C}$ for $3 \mathrm{~h}$. The reacting solutions were cooled down to room temperature and then purified by dialysis (14000 MWCO) in excessive fresh water changed several times per day to remove unreacted monomers and impurities. The as-prepared NHC solution was transferred into the cuvette, and then the hydrodynamic diameters of NHCs were measured by dynamic light scattering (DLS) instrument (Zetasizer Nano ZS, Malvern Instruments Ltd.) at $25{ }^{\circ} \mathrm{C}$. The lower critical solution temperature (LCST) was obtained from a series of as-prepared NHC hydrodynamic diameters measured at different temperatures. The concentration of hydrogel solution was calculated after lyophilisation of $5 \mathrm{~mL}$ polymer sample solution.

\section{Fabrication and characterization of nano hydrogel colloidal array}

The nano hydrogel colloidal array (NHCA) was fabricated via a vertical deposition method ${ }^{37}$ on a clean glass slide treated with a $\mathrm{H}_{2} \mathrm{SO}_{4}-\mathrm{H}_{2} \mathrm{O}_{2}$ solution. The glass slide was vertically dipped into a vial containing the NHC solutions $(0.3 \mathrm{wt} \%)$ at $30{ }^{\circ} \mathrm{C}$ under $50 \%$ relative humidity in an incubator for 3-4 days. After complete evaporation of water, NHCA were obtained on the glass slide.

Morphology images of the NHCA were obtained with a field emission scanning electron microscope (HITACHI S-4800, Japan) after sputter coating a layer of platinum on the surface of NHCA. Photographs of the as-prepared films on glass slide were taken with a digital camera. Reflection spectrum of the NHCA was recorded using a fiber optic UV-vis spectrometer (Avaspec-2048TEC, Avantes) with an AvaLight-DH-S-BAL light source and an AvaSpec-ULS-TEC fiber optic reflection probe.

\section{Humidity sensor response}

NHCA slides were placed in a hermetic container $(45 \mathrm{~cm} \times$ $45 \mathrm{~cm} \times 45 \mathrm{~cm})$. Humidity inside the container was controlled using a desiccator containing a large dish of a warm water or supersaturated salt solutions, ${ }^{\mathbf{3 0}}$ and a fan to gently circulate the air surrounding the measurement apparatus. Real-time relative humidity $(\mathrm{RH})$ and temperature values was monitored with a digital temperature \& humidity sensor (AS105, Guangzhou) probe inside the container after the equilibrium of $\mathrm{RH}$.

To study the response to relative humidity, reflection spectrum of the NHCA at an atmospheric condition or a controlled humidity condition was recorded. The NHCA response time was investigated when exposed to a certain relative humidity $(\mathrm{RH})$ from the atmosphere conditions of $\mathrm{RH} 20 \%$. Here, the $\mathrm{RH}$ considered in the experiment were $50 \%$ and $100 \%$ respectively. Reversibility of the NHCA was tested by raising and dropping humidity repeatedly.

\section{Results and discussion}

\section{Characterization of the nano hydrogel colloidal}

The monodispersed nano hydrogel colloidal (NHC) could be obtained while the backbone monomer (NIPAM), hydrophobic $(t$-BAM) and hydrophilic monomer (AAc) and the surfactant (SDS) reacted in appropriate ratios. NIPAM as the conventional thermosensitive monomer exhibits lower critical solution temperature (LCST) at $32-34{ }^{\circ} \mathrm{C}$. However, the LCST value of the NHC can be tuned by adjusting the hydrophilic-hydrophobic balance. The LCST value of the corresponding NHC with the formula NIPAM/AAc/t-BAM/BIS (38/20/40/2, mol\%) was confirmed as $11-12{ }^{\circ} \mathrm{C}$, which was proved by dynamic light scattering (Fig. 1). The lower LCST of NHC indicates that NHC is in a collapsed state above this temperature. That means the temperature-sensitive property of NHC would not interfere with 


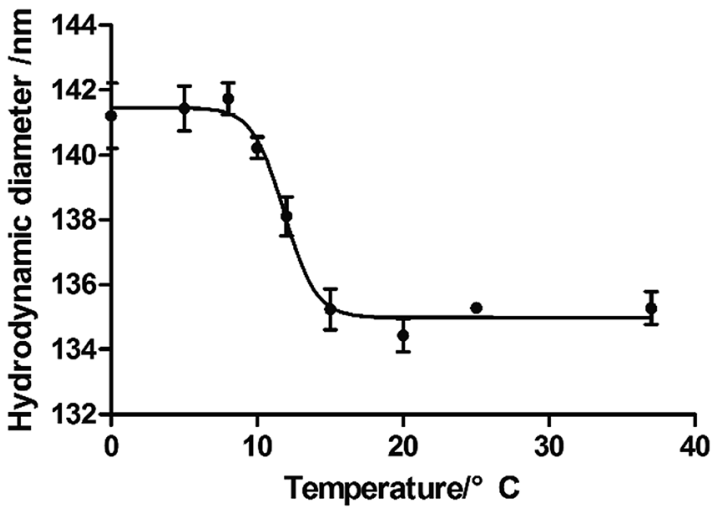

Fig. 1 The hydrodynamic diameters of the NHC with an optimised monomer molar ratio.

the swelling/shrinkage properties due to water absorption when measuring humidity at room temperature.

The hydrodynamic diameters of NHCs could be controlled by the concentration of surfactant SDS in pre-polymer solution. A series of NHCs with different particle size from 60-600 nm was obtained while the amount of SDS used in the polymerization process was adjusted from 0.02 to $0.1 \mathrm{mg} \mathrm{mL}^{-1}$, and the distribution trend of the colloidal size with SDS concentration is displayed in Fig. 2.

During the reaction, electrostatic repulsion force of colloids was enhanced when SDS concentration increased, which results in a shorter chain polymer, therefore, the smaller size of the nanoparticles was obtained. All the NHCs are monodispersed with uniform particle size while their PDI value was below 0.1 monitored by a Zetasizer Nano ZS DLS instrument in aqueous solutions.

\section{Characterization of the nanohydrogel colloidal array}

A series of 3D NHCA were self-assembled via the vertical deposition method $^{37}$ with the nano hydrogel colloidal solution, which concentration was confirmed as $6.0-8.1 \mathrm{mg} \mathrm{mL}^{-1}$ by a Gravimetric analysis after lyophilisation, and then was diluted

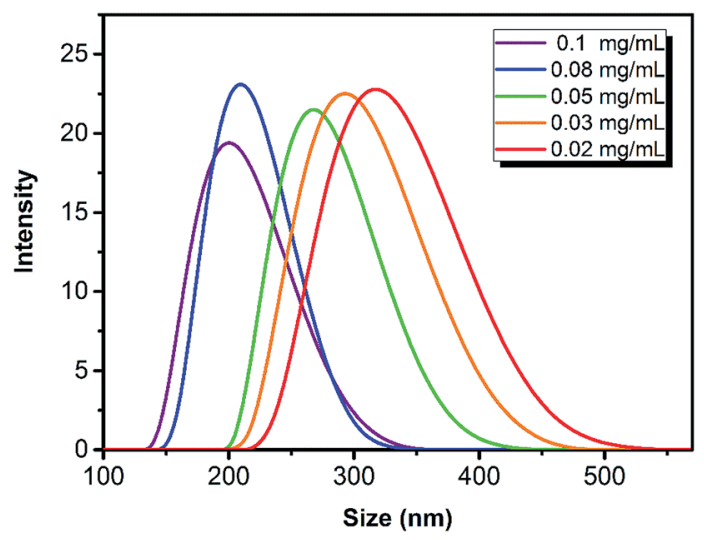

Fig. 2 DLS distributions of $\mathrm{NHC}$ with different sizes while the concentration of SDS used is from $0.1 \mathrm{mg} \mathrm{mL}^{-1}$ (purple) to $0.02 \mathrm{mg}$ $\mathrm{mL}^{-1}$ (red). to $3.0 \mathrm{mg} \mathrm{mL} \mathrm{m}^{-1}$ to prepare the array with a certain thickness. It is an easy, cost-effective and efficient approach for obtaining NHCA from NHCs. ${ }^{28}$ The uniform size and fine spherical shape of NHCs together with the regular microstructure of the asprepared NHCA were proved by the SEM image in Fig. 3(a). In addition, the self-assembly process is strict with the uniformity and sphericity of the colloidal crystals. ${ }^{38}$ The structured array also implies high uniform quality of the NHCs. From the cross view of the NHCA in Fig. 3(b), the thickness of typical asprepared NHCA is three micrometres with about 20 layers. Such a thin array makes the interface between the NHCA and the target larger, the response becomes faster.

Their transmission spectra was also proved by a fiber optic UV-vis spectrometer as presented in Fig. 4 . The inserts depict various structural colours of these as-prepared hydrogel colloidal PC shows visible colour from purple, blue, green, orange to red corresponding to the arrays with different particles in $169 \mathrm{~nm}, 186 \mathrm{~nm}, 214 \mathrm{~nm}, 230 \mathrm{~nm}$ and $285 \mathrm{~nm}$. Therefore, the PCs covering the full visible wavelength range $(400-760 \mathrm{~nm})$ could be obtained with different sizes of the NPs.

\section{Humidity response of the nano hydrogel colloidal array}

Due to the intrinsic humidity sensitivity of Aac used as the negative charged monomer in this study, the as-prepared NHCA shows sensitive to humidity. The reflection peak get red shift when the relative humidity (RH) increased. From the colour changes and the reflection peak shifts, the relative humidity could be clearly indicated. Herein, we give a typical example of the NHCA with a green structural colour prepared by nanoparticle of $230 \mathrm{~nm}$ responsing to the $\mathrm{RH}$ from $20 \%$ to $50 \%$ and $99.9 \%$, respectively.

It is seen from Fig. 5, both reflection peak wavelengths stay the same after a rapid red shift of reflection peak wavelength within equilibrium time of $10 \mathrm{~min}$. Compared with the traditional commercial hygrometers that can achieve the balance within 60 seconds, it takes time for sensors made from polymer material to deform when humidity changes. This time depends on the nature of the material itself. Three-dimensional ordered porous poly(ethylene glycol) hydrogel could expand in $1 \mathrm{~min}$ and reach stability after $70 \mathrm{~min},{ }^{39}$ while transition time of PAAm-P(St-MMA-AA) PC hydrogel between the dry and fully wetted states was less than 3 min. ${ }^{19}$ The NHCA was thin enough
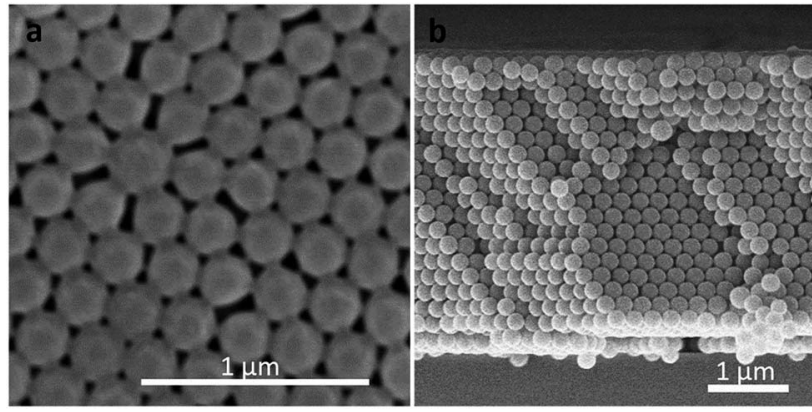

Fig. 3 SEM images of the NHCA with colloidal diameter of $214 \mathrm{~nm}$. (a) Top view. (b) Cross view. 


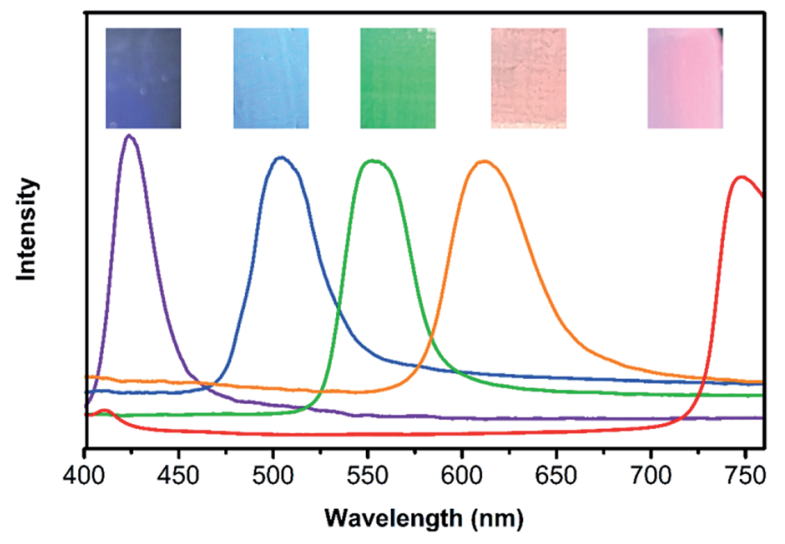

Fig. 4 UV-vis spectra of the NHCA with particle diameter of $169 \mathrm{~nm}$, $186 \mathrm{~nm}, 214 \mathrm{~nm}, 230 \mathrm{~nm}$ and $285 \mathrm{~nm}$. The inset shows the structure color of the as-prepared NHCA. The photos and data were taken at atmospheric conditions.

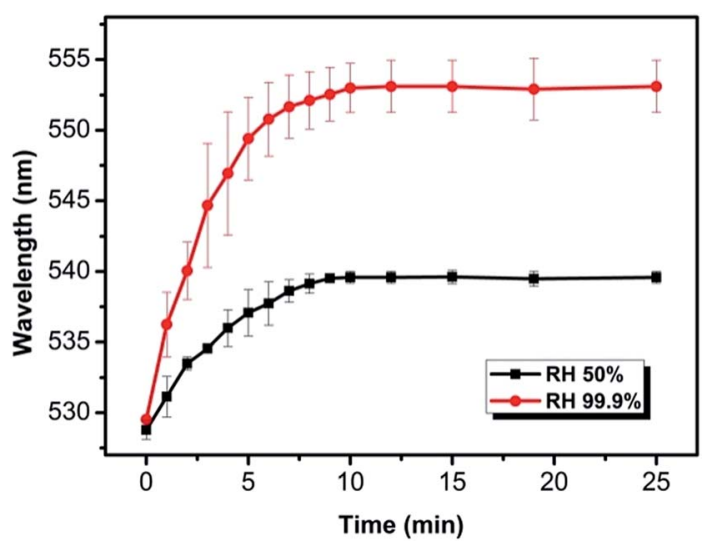

Fig. 5 Time dependence of the NHCA exposed to $50 \% \mathrm{RH}$ (black) and $100 \% \mathrm{RH}$ (red) from ambient $\mathrm{RH}$ of $20 \%$.

to achieve a somewhat short equilibrium time. Red shift of $24 \mathrm{~nm}$ and $11 \mathrm{~nm}$ was monitored during humidity increase in the black line with squares and red line with dots, correspondingly. The swelling of NHC contribute to the red shift of the reflection peak, and the swell ratio can be calculated by the reflection peak change. $(1):^{10,40}$

For the NHCA, the reflection obeys Bragg and Snell's law

$$
m \lambda_{\text {peak }}=2 d_{111}\left(n_{\mathrm{eff}}^{2}-\sin ^{2} \theta\right)^{1 / 2}
$$

where, $m$ is the order of reflection; $\lambda_{\text {peak }}$ is the wavelength of the reflected light; $n_{\text {eff }}$ is the mean effective refractive index; $\theta$ is the angle between the incident light and the normal to the reflection plane. $d_{111}$ is the lattice spacing between array planes which can be calculated by the diameter of NHC, defined as $D$ here, as shown in eqn (2).

$$
d_{111}=\sqrt{\frac{2}{3}} D
$$

In this study, the incident angle $\theta$ was fixed and the average value of effective refractive index $n_{\text {eff }}$ was constant. As for the first order diffraction, the value of $m$ is 1 . Therefore, we can get a linearity between the lattice spacing and the diameter $D$ in eqn (3).

$$
\lambda_{\text {peak }}=\alpha D
$$

Herein, the linear coefficient is defined as $\alpha$. The swelling ratio $R$ of the NHC in response to humidity could be calculated as eqn (4).

$$
R=\left(\lambda_{\text {peak }, 1}-\lambda_{\text {peak }, 0}\right) / \lambda_{\text {peak }, 0}
$$

where, $\lambda_{\text {peak,1 }}$ is the wavelength of the reflected light after adsorption equilibrium, $\lambda_{\text {peak, } 0}$ is the initial wavelength of the reflected light.

Therefore, the swelling ratios of the NHC is $4.5 \%$ and $2.1 \%$ respectively corresponding to eqn (4), indicating that NHC has the swelling properties. Compared with the common PC fabricated with solid colloids that need to be embedded with gel, NHCA is easy prepared, which makes it easier for use in the future.

After absorbing water, the structure of the network is swollen, which leads to the increase of the particle size and the shift of the reflection peak. The scheme was shown in Fig. 6. If more water is added, the particles will be dissolved. The PNIPAM exhibits an LCST of $32{ }^{\circ} \mathrm{C}$, while the LCST drops to 11$12{ }^{\circ} \mathrm{C}$ for NHC, which contains temperature-sensitive monomer NIPAM and other functional monomers such as acrylic acid. The NHC also has properties of hydrophilicity at low temperature and hydrophobicity at high temperature. Tanaka ${ }^{\mathbf{4 1}}$ suggested whether the polymer gel should absorb water depending on the combination of van der Waals forces, hydrogen bonding, electrostatic interaction and hydrophobic interaction. The condition temperature is higher than the LCST of NHC, which means the NHC is in a hydrophobic state. Therefore, we

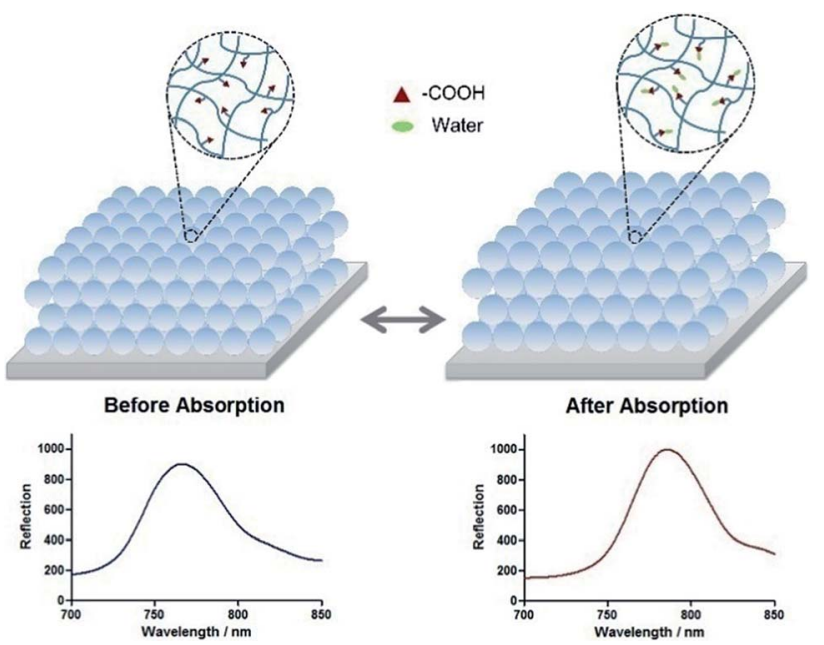

Fig. 6 Sketch diagram of the detection mechanism for the target by the NHCA. 


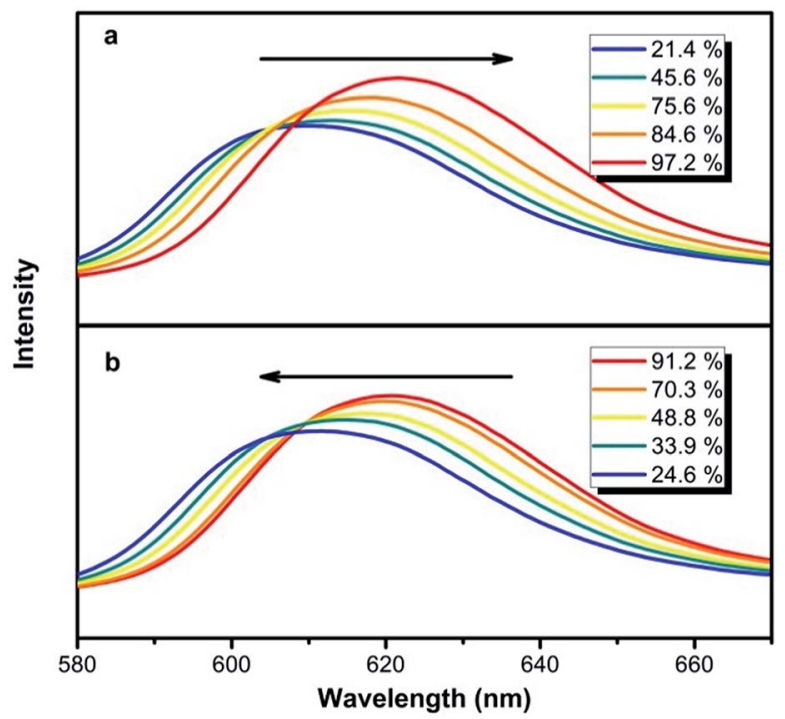

Fig. 7 UV-vis spectra of the as-prepared hydrogel colloidal PCs with humidity change. (a) Water absorption (b) water desorption. The NHCA were assembled from $\mathrm{NHC}$ of $230 \mathrm{~nm}$.

proposed that water absorption at NHCA is mainly due to the contribution of the acrylic acid component.

The shifts and intensity changes of reflection peak in response to humidity were monitored, which discloses the particle swelling behavior as shown in Fig. 7. A red shifts and intensity increase of reflection peak when increasing humidity and a blue shifts and intensity decrease of reflection peak when decreasing humidity was observed.

As the ambient humidity increases, the carboxyl groups in the polymer chains within the colloid formed a more stable bond with the water molecules. The initially dry hydrogel colloids swelled to a degree that the PC on the glass slide was close-packed and the compactness of the close-packed structure was strengthened until reaching equilibrium, and vice versa.

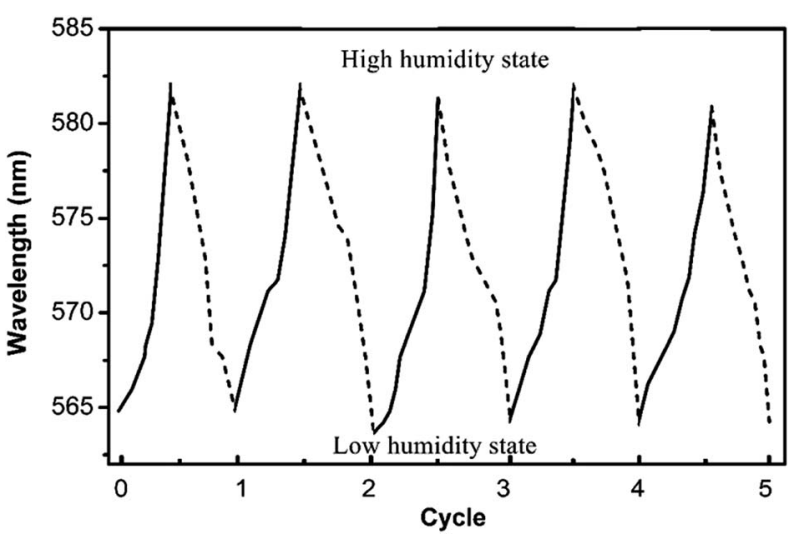

Fig. 8 Reversible wavelength changes of UV-vis reflection peak of the nanohydrogel colloidal PC with relative humidity by alternately exposing to increasing (the solid lines) and decreasing (the dashed lines) relative humidity conditions (between $20 \%$ and approaching $100 \%$ ).
The NHCA showed a reversible reflective wavelength shift according to the swelling and shrinkage behaviour of the NHC when the humidity changed, which makes it a potential tool to detect the humidity sensitively and quickly.

\section{Reversibility of the nano hydrogel colloidal array in response to humidity}

The NHCA fabricated from the soft nano-hydrogel particles is stable and reusable. The reversibility of the NHCA was evaluated on the humidity response by cycle method of humidity (Fig. 8). It was shown that the NHCA could retain its initial response to humidity after 5 rounds relative humidity recycles from $20 \%$ to $100 \%$, which means the sensor presents good stability and reversibility and can be used many times in humidity range.

\section{Conclusions}

Compared with the conventional photonic crystal, soft and uniform nano hydrogel colloids dispersed in water with biofriend, stable physical and chemical properties, were selfassembled to get a 3D-array. This nano hydrogel colloidal array with a bright structure color can be used as a humidity sensor. The response mechanism of the sensor is based on the changes in the compactness of close-packed structure and refractive index of the array of the hydrogel colloids, which can also be applied to biomolecules for response. Full wavelength coverage will meet different requirements. Further work on optimizing the performance to realize naked-eye detection as well as diminishing the response time is under way.

\section{Conflicts of interest}

There are no conflicts to declare.

\section{Acknowledgements}

Financial support from the Natural Science Foundation of China (No. 21375009) is highly appreciated.

\section{Notes and references}

1 G. Coviello, V. Finazzi, J. Villatoro and V. Pruneri, Thermally stabilized PCF-based sensor for temperature measurements up to $1000^{\circ} \mathrm{C}$, Opt. Express, 2009, 17(24), 21551-21559.

2 W. Qian, C. Zhao, S. He, X. Dong, S. Zhang, Z. Zhang, S. Jin, J. Guo and H. Wei, High-sensitivity temperature sensor based on an alcohol-filled photonic crystal fiber loop mirror, Opt. Lett., 2011, 36(9), 1548-1550.

3 N. Griffete, H. Frederich, A. Maître, M. M. Chehimi, S. Ravaine and C. Mangeney, Photonic crystal pH sensor containing a planar defect for fast and enhanced response, J. Mater. Chem., 2011, 21(34), 13052.

4 K. Lee and S. A. Asher, Photonic Crystal Chemical Sensors: pH and Ionic Strength, J. Am. Chem. Soc., 2000, 122(39), 9534-9537.

5 C. Ma, Y. Jiang, X. Yang, C. Wang, H. Li, F. Dong, B. Yang, $\mathrm{K}$. $\mathrm{Yu}$ and $\mathrm{Q}$. Lin, Centrifugation-induced water-tunable 
photonic colloidal crystals with narrow diffraction bandwidth and highly sensitive detection of SCN, ACS Appl. Mater. Interfaces, 2013, 5(6), 1990-1996.

6 M. Kamenjicki Maurer, I. K. Lednev and S. A. Asher, Photoswitchable Spirobenzopyran-Based Photochemically Controlled Photonic Crystals, Adv. Funct. Mater., 2005, 15(9), 1401-1406.

7 J. Hyon, C. Seo, I. Yoo, S. Song and Y. Kang, Glassy photonic inks encapsulated in core-shell microcapsules for local electric field sensors, Sens. Actuators, B, 2016, 223, 878-883.

8 L. M. Fortes, M. C. Gonçalves and R. M. Almeida, Flexible photonic crystals for strain sensing, Opt. Mater., 2011, 33(3), 408-412.

9 F. Liu, S. Huang, F. Xue, Y. Wang, Z. Meng and M. Xue, Detection of organophosphorus compounds using a molecularly imprinted photonic crystal, Biosens. Bioelectron., 2012, 32(1), 273-277.

10 F. Wang, Z. Zhu, M. Xue, F. Xue, Q. Wang, Z. Meng, W. Lu, W. Chen, F. Qi and Z. Yan, Cellulose photonic crystal film sensor for alcohols, Sens. Actuators, B, 2015, 220, 222-226.

11 J. B. Jensen, L. H. Pedersen, P. E. Hoiby, L. B. Nielsen, T. P. Hansen, J. R. Folkenberg, J. Riishede, D. Noordegraaf, K. Nielsen, A. Carlsen and A. Bjarklev, Photonic crystal fiber based evanescent-wave sensor for detection of biomolecules in aqueous solutions, Opt. Lett., 2004, 29(17), 1974-1976.

12 M. Ben-Moshe, V. L. Alexeev and S. A. Asher, Fast Responsive Crystalline Colloidal Array Photonic Crystal Glucose Sensors, Anal. Chem., 2006, 78(14), 5149-5157.

13 S. Pal, E. Guillermain, R. Sriram, B. L. Miller and P. M. Fauchet, Silicon photonic crystal nanocavity-coupled waveguides for error-corrected optical biosensing, Biosens. Bioelectron., 2011, 26(10), 4024-4031.

14 P.-S. Tsai, Y.-M. Yang and Y.-L. Lee, Fabrication of Hydrophobic Surfaces by Coupling of Langmuir-Blodgett Deposition and a Self-Assembled Monolayer, Langmuir, 2006, 22, 5660-5665.

15 H. Yang, P. Jiang and B. Jiang, Vapor detection enabled by self-assembled colloidal photonic crystals, J. Colloid Interface Sci., 2012, 370(1), 11-18.

16 C. Guo, C. Zhou, N. Sai, B. Ning, M. Liu, H. Chen and Z. Gao, Detection of bisphenol A using an opal photonic crystal sensor, Sens. Actuators, B, 2012, 166-167, 17-23.

17 X. Hong, Y. Peng, J. Bai, B. Ning, Y. Liu, Z. Zhou and Z. Gao, A Novel Opal Closest-Packing Photonic Crystal for Naked-Eye Glucose Detection, Small, 2014, 10(7), 1308-1313.

18 W.-K. Kuo, H.-P. Weng, J.-J. Hsu and H. H. Yu, A bioinspired color-changing polystyrene microarray as a rapid qualitative sensor for methanol and ethanol, Mater. Chem. Phys., 2016, 173, 285-290.

19 J. Wang, Y. Wen, H. Ge, Z. Sun, Y. Zheng, Y. Song and L. Jiang, Simple Fabrication of Full Color Colloidal Crystal Films with Tough Mechanical Strength, Macromol. Chem. Phys., 2006, 207(6), 596-604.

20 T. Dey, Colloidal crystalline array of hydrogel-coated silica nanoparticles: effect of temperature and core size on photonic properties, J. Sol-Gel Sci. Technol., 2010, 57(2), 132-141.

$21 \mathrm{X}$. Su, J. Chang, S. Wu, B. Tang and S. Zhang, Synthesis of highly uniform $\mathrm{Cu}_{2} \mathrm{O}$ spheres by a two-step approach and their assembly to form photonic crystals with a brilliant color, Nanoscale, 2016, 8(11), 6155-6161.

22 K. Jiang, D. Kuang, T. Fei and T. Zhang, Preparation of lithium-modified porous polymer for enhanced humidity sensitive properties, Sens. Actuators, B, 2014, 203, 752-758.

23 Q. Wang, J. Chang, W. Wei, C. Zhu and C. Tian, Dual-beam wavelength modulation spectroscopy for sensitive detection of water vapor, Appl. Phys. B, 2014, 117(4), 1015-1023.

24 J. D. Joannopoulos, P. R. Villeneuve and S. Fan, Photonic crystals: putting a new twist on light, Nature, 1997, 386(6621), 143-149.

25 C. López, Materials Aspects of Photonic Crystals, Adv. Mater., 2003, 15(15), 1679-1704.

26 C. I. Aguirre, E. Reguera and A. Stein, Tunable Colors in Opals and Inverse Opal Photonic Crystals, Adv. Funct. Mater., 2010, 20(16), 2565-2578.

27 B. Zhang, A. W. Morales, R. Peterson, L. Tang and J. Y. Ye, Label-free detection of cardiac troponin I with a photonic crystal biosensor, Biosens. Bioelectron., 2014, 58, 107-113.

$28 \mathrm{~J}$. Ge and Y. Yin, Responsive photonic crystals, Angew. Chem., Int. Ed., 2011, 50(7), 1492-1522.

29 Y. Zhao, Z. Xie, H. Gu, C. Zhu and Z. Gu, Bio-inspired variable structural color materials, Chem. Soc. Rev., 2012, 41(8), 3297-3317.

30 N. L. Smith, Z. Hong and S. A. Asher, Responsive Ionic Liquid Polymer 2D Photonic Crystal Gas Sensors, Analyst, 2014, 139, 6379.

31 R. A. Barry and P. Wiltzius, Humidity-Sensing Inverse Opal Hydrogels, Langmuir, 2006, 22(3), 1369-1374.

$32 \mathrm{H}$. Kawaguchi, Thermoresponsive microhydrogels: preparation, properties and applications, Polym. Int., 2014, 63(6), 925-932.

33 C. X. Ma, X. X. Le, X. L. Tang, J. He, P. Xiao, J. Zheng, H. Xiao, W. Lu, J. W. Zhang, Y. J. Huang and T. Chen, A Multiresponsive Anisotropic Hydrogel with Macroscopic 3D Complex Deformations, Adv. Funct. Mater., 2016, 26(47), 8670-8676.

$34 \mathrm{Y}$. Hoshino and K. J. Shea, The evolution of plastic antibodies, J. Mater. Chem., 2011, 21(11), 3517-3521.

35 J. M. Beierle, K. Yoshimatsu, B. Chou, M. A. Mathews, B. K. Lesel and K. J. Shea, Polymer nanoparticle hydrogels with autonomous affinity switching for the protection of proteins from thermal stress, Angew. Chem., Int. Ed., 2014, 53(35), 9275-9279.

36 K. Yoshimatsu, T. Yamazaki, Y. Hoshino, P. E. Rose, L. F. Epstein, L. P. Miranda, P. Tagari, J. M. Beierle, Y. Yonamine and K. J. Shea, Epitope discovery for a synthetic polymer nanoparticle: a new strategy for developing a peptide tag, J. Am. Chem. Soc., 2014, 136(4), 1194-1197.

37 S. Reculusa, P. Masse and S. Ravaine, Three-dimensional colloidal crystals with a well-defined architecture, J. Colloid Interface Sci., 2004, 279(2), 471-478. 
38 Q. Xu, Y. Lv, C. Dong, T. S. Sreeprased, A. Tian, H. Zhang, Y. Tang, Z. Yu and N. Li, Three-dimensional micro/ nanoscale architectures: fabrication and applications, Nanoscale, 2015, 7(25), 10883-10895.

39 B. Yu, H. Cong, Z. Yang, S. Yang, Y. Wang, F. Zhai and Y. Wang, Preparation of Humidity-Sensitive Poly(Ethylene Glycol) Inverse Opal Micropatterns Using Colloidal Lithography, Materials, 2017, 10(9), 1035.
40 T. Endo, Y. Yanagida and T. Hatsuzawa, Colorimetric detection of volatile organic compounds using a colloidal crystal-based chemical sensor for environmental applications, Sens. Actuators, B, 2007, 125(2), 589-595.

41 T. Tanaka, Phase transitions in gels and a single polymer, Polymer, 1979, 20(11), 1404-1412. 